\title{
Effects of short-term oral vitamin A supplementation on the ocular tear film in patients with dry eye
}

This article was published in the following Dove Medical Press journal: Clinical Ophthalmology

Saud A Alanazi
Gamal A El-Hiti

Abdulaziz A Al-Baloud

Mohamed I Alfarhan

Ammar Al-Shahrani

Abdulkareem A Albakri

Saad Alqahtani

Ali M Masmali

Cornea Research Chair, Department of Optometry, College of Applied

Medical Sciences, King Saud

University, Riyadh I I433, Saudi Arabia
Correspondence: Saud A Alanazi; Gamal A El-Hiti

Department of Optometry, College of Applied Medical Sciences, King Saud University, PO Box 10219, Riyadh II433, Saudi Arabia

Tel +966 II $4693543 ;+966$ | | 4693778

Email saaalanazi@ksu.edu.sa;

gelhiti@ksu.edu.sa
Objective: To investigate the effects of short-term oral vitamin A supplementation on the ocular tear film in patients with dry eye.

Methods: In total, 30 male patients with dry eye (age range, 18-38 years; mean age, $25.2 \pm 2.8$ years) who did not wear contact lenses or exhibit any ocular (other than dry eye) or systemic diseases were included, along with 30 age-matched men (control group; mean age, $24.5 \pm 2.3$ years) with healthy eyes. Subject exclusion was based on the findings of the McMonnies questionnaire (cutoff score for dry eye: 14.5) and slit-lamp biomicroscopy. All subjects received an oral vitamin A supplement at a daily dose of $1,500 \mathrm{mg}$ for 3 consecutive days. The phenol red thread (PRT) test was performed along with assessments of tear ferning (TF), tear osmolarity, and the tear break-up time (TBUT) before and 24 hours after the third dose of the vitamin A supplement. A 10-minute interval was observed between different tests.

Results: In the dry eye group, the TF grade (Wilcoxon test, $P=0.01$ ) exhibited a significant decrease, while the tear osmolarity value ( $t$-test, $P=0.01)$ exhibited a significant increase after vitamin A supplementation. The PRT test findings $(P=0.17)$ and TBUT $(P=0.49)$ showed no significant differences before and after vitamin A supplementation. In the control group, vitamin A supplementation showed no significant effects on TF $(P=0.74)$, tear osmolarity $(P=0.55)$, the TBUT $(P=0.19)$, and the PRT test scores $(P=0.48)$.

Conclusion: Our findings suggest that short-term oral vitamin A supplementation improves the quality, but not quantity, of tears in patients with dry eye. Future studies should involve larger patient samples and longer periods of vitamin A supplementation.

Keywords: vitamin A supplement, dry eye, tear film, tear ferning, phenol red thread test, tear break-up time, tear osmolarity

\section{Introduction}

Vitamin A is a mixture of retinol, retinal, and retinoic acid (Figure 1) along with other components such as $\beta$-carotene. ${ }^{1}$ The most common animal sources for vitamin $A$ are cod liver oil, liver, butter, cheese, eggs, and fish, whereas the most common vegetable sources include sweet potato, carrot, broccoli, sweet red pepper, spinach, and lettuce. Retinol and carotene are the most common forms of vitamin A in food. ${ }^{2}$ Vitamin A is essential for good vision, growth, reproduction, bone metabolism, gene transcription, and immune health. ${ }^{3}$ Moreover, vitamin $\mathrm{A}$ in the form of retinal combines with opsin to produce rhodopsin in the retina, which is important for both color and scotopic (low-light) vision. ${ }^{4}$

Vitamin A deficiency is very common in children aged $<5$ years. It affects around a third of the pediatric population worldwide and causes blindness in 250,000-500,000 children in developing countries each year. ${ }^{5,6}$ A previous study found that a diet containing low levels of vitamin A was found to be the main cause for night blindness 


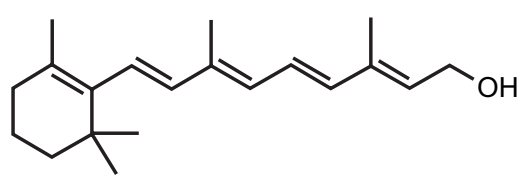

Retinol

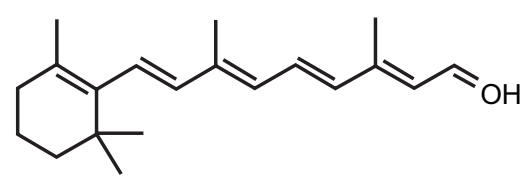

Retinal

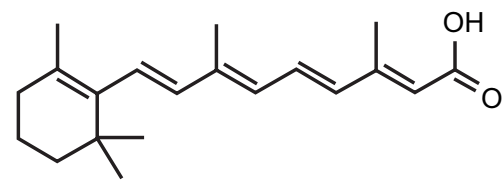

Retinoic acid

Figure I Chemical structure of vitamin A.

among soldiers in the American civil war. ${ }^{7}$ Vitamin A deficiency can also lead to corneal dryness (xerosis), keratomalacia, and corneal ulceration, which are very common in children with poor diets. ${ }^{8}$ The deficiency can occur as a primary or secondary deficiency. The former type is common in adults and children with an inadequate intake of vegetables, fruits, and dairy products, while the second type is associated with chronic alcoholism, smoking, and malabsorption of lipids. Furthermore, zinc is necessary for the metabolism of vitamin $\mathrm{A}$, and its low uptake can lead to vitamin A deficiency. ${ }^{9}$ A previous study found that a supplement containing vitamin A along with zinc could lower the risk of malaria and fever in African children. ${ }^{9}$ Therefore, children should essentially receive a nutritional diet that contains adequate amounts of vitamin $\mathrm{A}$ in order to avoid health problems. ${ }^{10,11}$

Tear film stability is very important for eyes to remain healthy and moist and to prevent dryness. ${ }^{12-14}$ The tear film consists of mucin, water, and lipid layers; ${ }^{15,16}$ however, its precise structure, function, and biophysical and biochemical properties are complex. ${ }^{17-20}$ Early detection of any tear film instability is necessary for maintaining the patient's quality of life and preventing dryness-related discomfort. Although a variety of diagnostic methods for dry eye are available, some of them are not suitable for clinical use. ${ }^{21}$ Dry eye should ideally be diagnosed using a combination of various tests rather than any single test. Measurement of the tear break-up time (TBUT) is one of the most common diagnostic tests for evaluation of the tear film quality. ${ }^{22}$ Meanwhile, assessment of tear film osmolarity provides information about tear dynamics and hyperosmolarity. ${ }^{23}$ The tear ferning (TF) test is also an effective technique for assessing the quality of tears and diagnosing eye dryness. ${ }^{24,25}$ The tear volume is most commonly measured using the Schirmer test and/or the phenol red thread (PRT) test. ${ }^{26}$ In addition, evaluation of tear evaporation rate is an efficient technique to detect dry eye syndrome. ${ }^{27}$ Dry eye-related questionnaires such as the McMonnies questionnaire and Ocular Surface Disease Index can be additionally used with all the above-mentioned tests. ${ }^{14}$ In the present study, we investigated the effects of short-term oral vitamin A supplementation on the quantity and quality of tears in patients with dry eye as a continuation of our extensive research on the human tear film..$^{28-34}$ The TF test based on the five-point grading scale ${ }^{24}$ was used for the first time to assess the effect of short-term vitamin A supplement on the quality of tears.

\section{Materials and methods Subjects}

This study included 30 male patients with dry eye (age range, 18-38 years; mean age, 25.2 \pm 2.8 years) who did not wear contact lenses or exhibit any systemic or ocular diseases other than dry eye. A control group including 30 age-matched men (mean age, $24.5 \pm 2.3$ years) with healthy eyes was also recruited. The exclusion criteria for the control group included smokers, diabetes, and subjects who had recent ocular surgeries, consumed medication, and abnormalities of the eyelids and lashes. A slit lamp was used to examine the external and anterior portions of the eye in order to rule out ocular diseases, while the McMonnies questionnaire was used to evaluate dry eye; the cutoff score for dry eye was 14.5. All subjects received a vitamin A supplement orally at a daily fixed dose of 1,500 mg (5,000 IU) for 3 consecutive days, ${ }^{35}$ between 8:00 and 9:00 am. Such a dose is considered acceptable by the National Health Institute. ${ }^{36}$ Vitamin A tablets were purchased from Solgar UK Ltd. (Aldbury, Hertfordshire, UK). The PRT test was performed along with assessments of TF, tear osmolarity, and TBUT before and 24 hours after the third dose of the vitamin A supplement. The tear sample was collected prior to the PRT test to avoid bias, followed by tear osmolarity test and finally the TBUT test was conducted. A 10-minute interval was observed between the different tests. ${ }^{30}$ This study followed the tenets of the Declaration of Helsinki, and written informed consent for study participation was obtained from all subjects after an explanation about the nature of the research was provided. Ethical approval was obtained from the Ethics Committee at the College of Applied Medical Sciences, King Saud University.

\section{PRT test}

The PRT test was performed once for the left eye of all subjects. A sterile cotton thread treated with the $\mathrm{pH}$ indicator 
phenol red was inserted in the lower lid and removed after 15 seconds for measurement of the red portion. The cutoff value for dry eye was $<10 \mathrm{~mm}^{28}$

\section{TF test}

The TF test was performed one time for the left eye. A tear sample $(1 \mu \mathrm{L})$ was collected from the lower meniscus of the eye using a glass capillary tube $(10 \mu \mathrm{L})$ and was dried on a glass slide for 10 minutes $\left(23^{\circ} \mathrm{C},<40 \%\right.$ humidity $)$. Tear ferns were observed under a digital microscope (Olympus DP72, ×10 magnification; Olympus Optical Co., Ltd., Tokyo, Japan) and TF grades were assigned. ${ }^{24}$

\section{Tear osmolarity test}

Tear osmolarity was measured once for the left eye using the TearLab ${ }^{\text {TM }}$ osmolarity system (TearLab ${ }^{\text {TM }}$ Corp., San Diego, CA, USA). A value of 298-302 mOsm/L was considered normal. $^{29}$

\section{TBUT test}

TBUT was measured to assess the tear film stability. Fluorescein was instilled into the subject's left eye, and the subject was instructed to refrain from blinking while the tear film was observed. A yellow barrier filter was used to enhance the visibility of the tear film break-up. TBUT was recorded as the number of seconds that elapsed between the last blink and the appearance of the first dry spot in the tear film. The measurement was performed in both eyes three times, and the average time was recorded. The cutoff value for dry eye is $<10$ seconds. ${ }^{37}$

\section{Statistical analysis}

All collected data were recorded in Microsoft Excel 2010 (Microsoft Office, Microsoft Corp., Redmond, WA, USA) and statistically analyzed using SPSS software, version 22 (IBM, Armonk, NY, USA).

\section{Results}

TF and PRT data for the dry eye group were not normally distributed (Kolmogorov-Smirnov test, $P<0.05$ ) and are presented as medians (interquartile ranges). In contrast, McMonnies scores, and osmolarity and TBUT values showed normal distribution (Kolmogorov-Smirnov test, $P>0.05$ ) and are presented as mean \pm SDs. In the control group, TF and osmolarity data were normally distributed (KolmogorovSmirnov test, $P>0.05$ ), while McMonnies scores and values for the PRT test and TBUT were not normally distributed (Kolmogorov-Smirnov test, $P<0.05$ ). The TF grades, PRT,
Table I TF grades, PRT test results, TBUT values, and osmolarity values for patients with dry eye who received short-term vitamin A supplementation

\begin{tabular}{l|l|l}
\hline Test & Before vitamin A & After vitamin A \\
\hline TF* & $2.4(0.5)$ & $1.4(\mathrm{I} . \mathrm{I})$ \\
PRT $(\mathrm{mm})$ & $27.9(5.8)$ & $30.7(4.2)$ \\
TBUT $(\mathrm{s})$ & $7.8 \pm 3.3$ & $8.8 \pm 4.5$ \\
Osmolarity* (mOsm/L) & $293.0 \pm 8.9$ & $303.0 \pm 9.3$ \\
\hline
\end{tabular}

Notes: *Statistically significant difference $(P<0.05)$ between pre- and postsupplementation values. TF and PRT test values are presented as medians (interquartile ranges), while TBUT and osmolarity values are presented as mean \pm SD.

Abbreviations: PRT, phenol red thread; TBUT, tear break-up time; TF, tear ferning.

TBUT, and osmolarity values for the study and control groups are shown in Tables 1 and 2, respectively.

The McMonnies scores for the study and control groups were $14.3 \pm 5.5$ and 6.0 (2.5), respectively. After vitamin A supplementation in the dry eye group, the TF grade was significantly decreased (before vs after: 2.4 [0.5] vs 1.4 [1.1]; Wilcoxon test, $P=0.01$ ), while the tear osmolarity value was significantly increased (before vs after: 293.0 \pm 8.9 vs $303.0 \pm 9.3$; $t$-test, $P=0.01)$. Although the PRT test and TBUT values were higher after supplementation than before supplementation, the differences were not significant (PRT test: Wilcoxon test, $P=0.17$; TBUT: $t$-test, $P=0.49$ ). In the control group, there were no significant differences in the TF grade $(P=0.74)$, tear osmolarity value $(P=0.55)$, PRT test result $(P=0.48)$, and TBUT value $(P=0.18)$ before and after supplementation. Figures 2 and 3 show representative images of TF obtained before and after vitamin A supplementation for a patient with dry eye and a patient with healthy eyes, respectively, while Figures 4 and 5 show side-by-side box plots for the TF grades and tear osmolarity values, respectively, before and after vitamin A supplementation in the dry eye group.

\section{Discussion}

Dry eye is a complex disease with various causes, and its severity is associated with several factors. ${ }^{38}$ Vitamins are

Table 2 TF grades, PRT test results, TBUT values, and osmolarity values for individuals with healthy eyes who received short-term vitamin A supplementation

\begin{tabular}{l|l|l}
\hline Test & Before vitamin A & After vitamin A \\
\hline TF & $1.2 \pm 0.5$ & $1.3 \pm 0.5$ \\
PRT $(\mathrm{mm})$ & $30.0(3.5)$ & $30.0(3.8)$ \\
TBUT $(\mathrm{s})$ & $7.0(7.5)$ & $8.5(7.8)$ \\
Osmolarity (mOsm/L) & $306.0 \pm \mathrm{I} 1.9$ & $308.0 \pm 15.4$ \\
\hline
\end{tabular}

Notes: TBUT and PRT test values are presented as medians (interquartile ranges), while TF and osmolarity values are presented as mean \pm SD.

Abbreviations: PRT, phenol red thread; TBUT, tear break-up time; TF, tear ferning. 


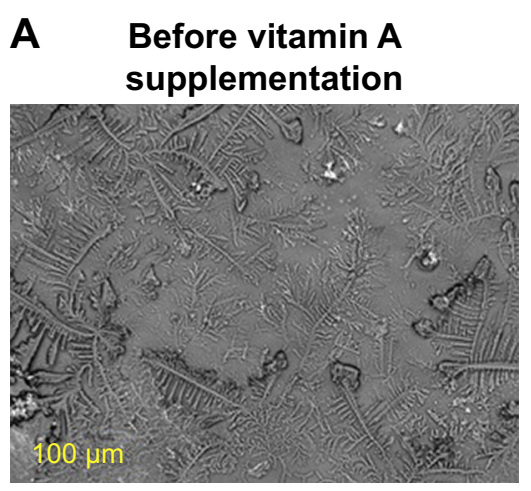

\section{B After vitamin A supplementation}

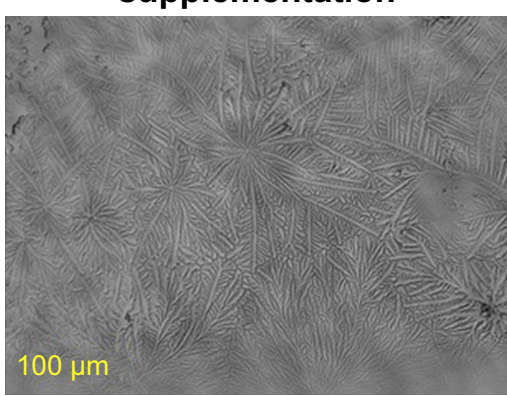

Figure 2 Images of tear ferning (TF) in the left eye that were obtained before and 24 hours after 3-day vitamin A supplementation for a patient with dry eye Notes: (A) before vitamin A supplementation; (B) after vitamin A supplementation.

\section{A Before vitamin A supplementation}

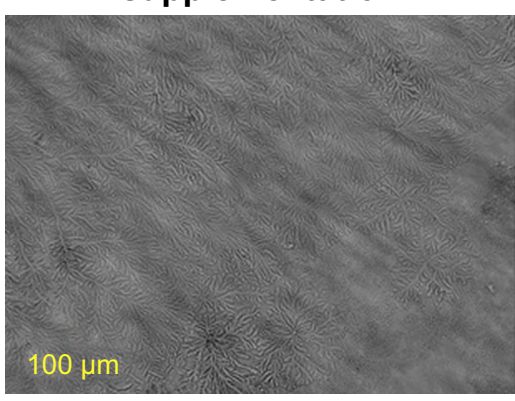

B After vitamin A supplementation

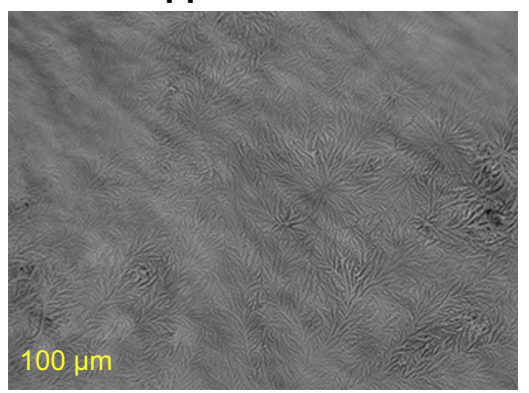

Figure 3 Images of tear ferning (TF) in the left eye that were obtained before and after vitamin A supplementation for an individual with healthy eyes. Notes: (A) before vitamin A supplementation; (B) after vitamin A supplementation.

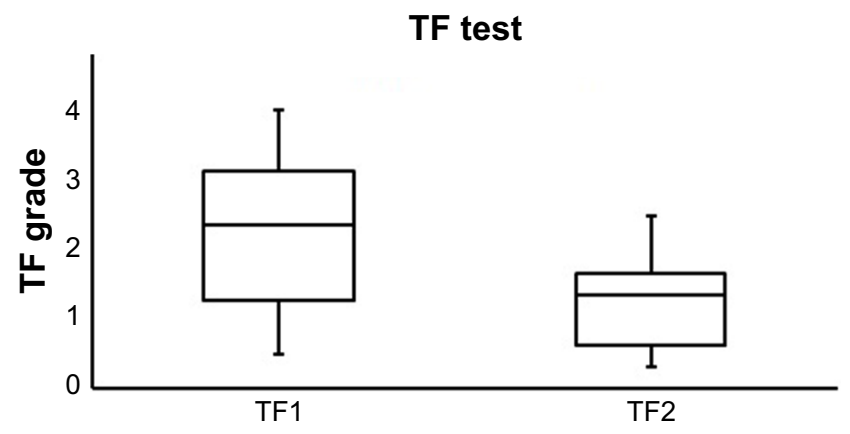

Figure 4 A side-by-side boxplot for the TF grades before (TFI) and after (TF2) 3-day vitamin A supplementation for patients with dry eye. Note: Statistically significant value at $P<0.05$.

Abbreviation: TF, tear ferning.

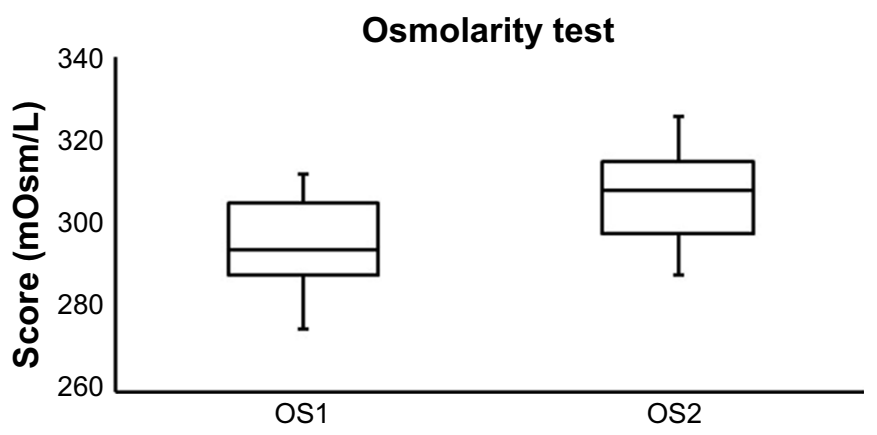

Figure 5 A side-by-side boxplot for the tear osmolarity values before (OSI) and after (OS2) vitamin A supplementation for patients with dry eye. Note: Statistically significant value at $P<0.05$. 
important for humans, and their deficiencies can lead to various complications, one of them being dry eye. For example, vitamin A can prevent night blindness, exophthalmia, and dry eye. ${ }^{39,40}$ In developing countries, vitamin A deficiency is the main cause for blindness among children. ${ }^{41}$ Therefore, in the present study, we examined the effects of short-term oral vitamin A supplementation on the ocular tear film in patients with dry eye in order to increase awareness about the importance of vitamin A for this patient population. We found that TF grades and tear osmolarity values improved after vitamin A supplementation in most patients with dry eye, whereas PRT test results and TBUT showed no significant changes. These results indicated that vitamin A supplementation is important for maintaining the health of the ocular tear film and can minimize the symptoms of dry eye.

Various studies have suggested an association between eye dryness and vitamin A deficiency. ${ }^{7,11,42}$ Vitamin A is an important component for the visual pigments and the integrity of the ocular surface, ${ }^{41}$ and it is used in the treatment of peripheral vision loss, age-related macular degeneration, retinitis pigmentosa, and superior limbic keratoconjunctivitis. ${ }^{43}$ Dry eye disease can result from vitamin A deficiency, with mild deficiency resulting in conjunctival changes and severe deficiency resulting in xerophthalmia. ${ }^{44} \mathrm{~A}$ healthy and balanced diet contributes to the treatment of such diseases. ${ }^{44}$ In one study comparing orally consumed vitamin A supplements and topically applied vitamin A eye drops for the management of eye dryness, the former were found to last for a longer duration, ${ }^{45}$ and the authors suggested that oral supplements are more effective in the treatment of dry eye syndrome than are commercially available lubricants. ${ }^{45}$ However, topical vitamin A can be used to regulate the proliferation of corneal epithelial cells, ${ }^{43}$ and topical lubricants have been used for the treatment of keratoconjunctivitis sicca, with a previous study showing a remarkable improvement after 3 months of treatment in $81 \%$ of the subjects $(n=17) .{ }^{46}$ In another study, topical trans-retinoic acid was found to be effective in the treatment of keratoconjunctivitis sicca. ${ }^{47}$ Thus, vitamin A not only ameliorates the symptoms of dry eye but also enhances the smoothness and stability of the ocular tear film and supports tear production. ${ }^{45,48}$ As opposed to the above-mentioned studies, one study showed that oral omega-3 supplements were more effective than were oral vitamin A supplements for the management of dry eye syndrome. ${ }^{49}$ Moreover, it has been shown that some eye drops cause allergy and last only for a short period of time. ${ }^{50}$ However, preservative-free vitamin A drops are more effective than other eye drops in the management of dry eye symptoms. ${ }^{44}$ Finally, vitamin A supplementation for 2 months was also found to be very effective in improving the visual acuity of both eyes and eliminating excessive tearing $;{ }^{51}$ therefore, patients with itchy eyes should review their dietary history to ensure adequate intake of vitamin $\mathrm{A} .{ }^{51}$

\section{Limitations}

This study has some limitations. First, the sample size was small, which makes it difficult to generalize the study findings. Second, the supplements were taken at a fixed dose for only 3 days. Further large-scale studies with longer periods of supplementation are required to support the role of vitamin A supplementation in the stability of the ocular tear film in patients with dry eye.

\section{Conclusion}

The findings of the present study suggest that short-term oral vitamin A supplementation improves the quality, but not quantity, of tears in patients with dry eye. Thus, oral vitamin A supplementation is important for maintaining the health of the ocular tear film and can minimize the symptoms of dry eye.

\section{Acknowledgment}

This project was supported by King Saud University, Deanship of Scientific Research, Research Chairs.

\section{Disclosure}

The authors report no conflicts of interest in this work.

\section{References}

1. Damodaran S, Parkin KL. Fennema's Food Chemistry. 5th ed. Boca Raton, FL: CRC Press; 2017.

2. de Man J. Principles of Food Chemistry. 3rd ed. Maryland: Aspen Publication Inc; 1999.

3. Tanumihardjo SA. Vitamin A: biomarkers of nutrition for development. Am J Clin Nutr. 2011;94(2):658S-665S.

4. US National Institutes of Health. Vitamin A. Bethesda, MA: Office of Dietary Supplements, US National Institutes of Health; 2016.

5. Black RE, Allen LH, Bhutta ZA, et al. Maternal and child undernutrition: global and regional exposures and health consequences. Lancet. 2008;371(9608):243-260.

6. Sommer A. Vitamin A deficiency and clinical disease: an historical overview. J Nutr. 2008;138(10):1835-1839.

7. Lanska DJ. Vitamin A-deficiency eye disease among soldiers in the U.S. civil war: spectrum of clinical disease. Mil Med.2015;180(7):774-779.

8. Sommer A, West KP. Vitamin A Deficiency: Health, Survival and Vision. New York: Oxford University Press; 1995.

9. Zeba AN, Sorgho H, Rouamba N, et al. Major reduction of malaria morbidity with combined vitamin A and zinc supplementation in young children in Burkina Faso: a randomized double blind trial. Nutr J. 2008;7(1):7.

10. Sommer A, Hussaini G, Tarwotjo I, Susanto D. Increased mortality in children with mild vitamin A deficiency. Lancet. 1983;322(8350):585-588.

11. Gilbert C. The eye signs of vitamin A deficiency. Community Eye Health. 2013;26(84):66-67. 
12. Rolando M, Zierhut M. The ocular surface and tear film and their dysfunction in dry eye disease. Surv Ophthalmol. 2001;45(Suppl 2): S203-S210.

13. Fenner BJ, Tong L. More to stable tears than thickness of the tear film lipid layer. Invest Ophthalmol Vis Sci. 2015;56(3):1601.

14. Bron AJ. Methodologies to diagnose and monitor dry eye disease: report of the diagnostic methodology Subcommittee of the International dry eye workshop (2007). Ocul Surf. 2007;5(2):108-152.

15. Khanal S, Millar TJ. Nanoscale phase dynamics of the normal tear film. Nanomedicine. 2010;6(6):707-713.

16. Tragoulias ST, Anderton PJ, Dennis GR, Miano F, Millar TJ. Surface pressure measurements of human tears and individual tear film components indicate that proteins are major contributors to the surface pressure. Cornea. 2005;24(2):189-200.

17. Cwiklik L. Tear film lipid layer: a molecular level view. Biochim Biophys Acta. 1858;2016(10):2421-2430.

18. Georgiev GA, Eftimov P, Yokoi N. Structure-function relationship of tear film lipid layer: A contemporary perspective. Exp Eye Res. 2017; 163:17-28.

19. Wizert A, Iskander DR, Cwiklik L. Organization of lipids in the tear film: a molecular-level view. PLoS One. 2014;9(3):e92461.

20. Sweeney DF, Millar TJ, Raju SR. Tear film stability: a review. Exp Eye Res. 2013;117:28-38.

21. Ay Y, Lu T, Pan AP, et al. Assessment of tear film optical quality dynamics. Invest Ophthalmol Vis Sci. 2016;57(8):3821-3827.

22. Smith J, Nichols KK, Baldwin EK. Current patterns in the use of diagnostic tests in dry eye Evaluation. Cornea. 2008;27(6):656-662.

23. Potvin R, Makari S, Rapuano C. Tear film osmolarity and dry eye disease: a review of the literature. Clin Ophthalmol. 2015;9:2039-2047.

24. Masmali AM, Murphy PJ, Purslow C. Development of a new grading scale for tear ferning. Cont Lens Anterior Eye. 2014;37(3):178-184.

25. Nebbioso M, Sacchetti M, Bianchi G, et al. Tear ferning test and pathological effects on ocular surface before and after topical cyclosporine in vernal keratoconjunctivitis patients. J Ophthalmol. 2018;2018(3):1-11.

26. Vitali C, Bombardieri S, Jonsson R, et al. European Study Group on Classification Criteria for Sjögren's Syndrome. Classification criteria for Sjögren's syndrome: a revised version of the European criteria proposed by the American-European Consensus Group. Ann Rheum Dis. 2002;61(6):554-558.

27. Abusharaha A, Alturki AA, Alanazi SA, et al. An assessment of the tear evaporation rate in thyroid gland patients. Clin Ophthalmol. 2019; 13:131-135.

28. Masmali A, Alqahtani TA, Alharbi A, El-Hiti GA. Comparative study of repeatability of phenol red thread test versus Schirmer test in normal adults in Saudi Arabia. Eye Contact Lens. 2014;40(3):127-131.

29. Masmali A, Alrabiah S, Alharbi A, El-Hiti GA, Almubrad T. Investigation of tear osmolarity using the TearLab osmolarity system in normal adults in Saudi Arabia. Eye Contact Lens. 2014;40(2):74-78.

30. Masmali AM, Al-Bahlal JM, El-Hiti GA, et al. Repeatability and diurnal variation of tear ferning test. Eye Contact Lens. 2015;41(5):262-267.

31. Masmali AM, Al-Qhtani S, Al-Gasham TM, El-Hiti GA, Purslow C, Murphy PJ. Application of a new grading scale for tear ferning in non-dry eye and dry eye subjects. Cont Lens Anterior Eye. 2015;38(1): $39-43$.

Clinical Ophthalmology

\section{Publish your work in this journal}

Clinical Ophthalmology is an international, peer-reviewed journal covering all subspecialties within ophthalmology. Key topics include: Optometry; Visual science; Pharmacology and drug therapy in eye diseases; Basic Sciences; Primary and Secondary eye care; Patient Safety and Quality of Care Improvements. This journal is indexed on Submit your manuscript here: http://www.dovepress.com/clinical-ophthalmology-journal
32. Saad AS, El-Hiti GA, Masmali AM. A computer-based image analysis for tear ferning featuring. J Innov Opt Health Sci. 2015;08(05):1550015.

33. Masmali AM, Al-Shehri A, Alanazi SA, Abusharaha A, Fagehi R, El-Hiti GA. Assessment of tear film quality among smokers using tear ferning patterns. J Ophthalmol. 2016;2016(4):1-5.

34. Masmali AM, Maeni YA, El-Hiti GA, Murphy PJ, Almubrad T. Investigation of ocular tear ferning in controlled and uncontrolled diabetic subjects. Eye Contact Lens. 2018;44(Suppl 2):S70-S75.

35. Chen C, Mistry G, Jensen B, et al. Pharmacokinetics of retinoids in women after meal consumption or vitamin A supplementation. J Clin Pharmacol. 1996;36(9):799-808.

36. National Institute of Health (NHS). Strengthening knowledge and understanding of dietary supplements. Available from: https://ods.od.nih. gov/factsheets/VitaminA-HealthProfessional/. Accessed October 21, 2018.

37. Lemp MA, Hamill JR. Factors affecting tear film breakup in normal eyes. Arch Ophthalmol. 1973;89(2):103-105.

38. The definition and classification of dry eye disease: report of the definition and classification Subcommittee of the International dry eye workshop (2007). Ocul Surf. 2007;5(2):75-92.

39. Mares JA, La Rowe TL, Blodi BA. Doctor, what vitamins should I take for my eyes? Arch Ophthalmol. 2004;122(4):628-635.

40. Lee MH, Sarossy MG, Zamir E. Vitamin A Deficiency Presenting with "Itchy Eyes". Case Rep Ophthalmol. 2015;6(3):427-434.

41. Al-Ani A, Ramaesh K, Wykes W. Diagnosis of vitamin A deficiency from its ocular manifestations in a British patient. Can J Ophthalmol. 2009;44(5):e43.

42. Faustino JF, Ribeiro-Silva A, Dalto RF, et al. Vitamin A and the eye: an old tale for modern times. Arq Bras Oftalmol. 2016;79(1):56-61.

43. Azuma M, Yabuta C, Fraunfelder FW, Shearer TR. Dry eye in LASIK patients. BMC Res Notes. 2014;7(1):420.

44. Stewart JM, Lee O-T, Wong FF, Schultz DS, Lamy R. Cross-linking with ultraviolet-A and riboflavin reduces corneal permeability. Invest Ophthalmol Vis Sci. 2011;52(12):9275-9278.

45. Ziada HEA. Oral vitamin-A including antioxidant formula versus topical vitamin A added to lubricant eye drops in treatment of dry eye syndrome; a comparative study. Int J Ophthalmol Res. 2017;3(4):252-258.

46. Soparkar CN, Wilhelmus KR, Koch DD, Wallace GW, Jones DB. Acute and chronic conjunctivitis due to over-the-counter ophthalmic decongestants. Arch Ophthalmol. 1997;115(1):34-38.

47. Galora A, Zheng DD, Arheart KL, et al. Dry eye medication use and expenditures: data from the medical expenditure panel survey 2000 to 2006. Cornea. 2012;31(12):1403-1407.

48. Ragam A, Kolomeyer AM, Kim JS, et al. Topical cyclosporine a 1\% for the treatment of chronic ocular surface inflammation. Eye contact Lens. 2014;40(5):283-288.

49. Foulks GN. Pharmacological management of dry eye in the elderly patient. Drugs Aging. 2008;25(2):105-118.

50. Bhargava R, Kumar P, Kumar M, Mehra N, Mishra A. A randomized controlled trial of omega-3 fatty acids in dry eye syndrome. Int $J$ Ophthalmol. 2013;6(6):811-816.

51. Lee MH, Sarossy MG, Zamir E. Vitamin A deficiency presenting with “itchy eyes". Case Rep Ophthalmol. 2015;6(3):427-434.
PubMed Central and CAS, and is the official journal of The Society of Clinical Ophthalmology (SCO). The manuscript management system is completely online and includes a very quick and fair peer-review system, which is all easy to use. Visit http://www.dovepress.com/ testimonials.php to read real quotes from published authors. 